\title{
Relapsed anaplastic lymphoma kinase-positive large B-cell lymphoma expressed cluster of differentiation 4 and cytokeratin: An initially misdiagnosed case corrected by immunoglobulin $\kappa$ locus gene rearrangement detection
}

\author{
YUNFEI SHI ${ }^{1}$, XIANGHONG LI ${ }^{1}$, YUQIN SONG ${ }^{2}$, LIXIN ZHOU ${ }^{1}$, QIN FENG ${ }^{1}$, \\ PING WANG $^{1}$, CHEN ZHANG $^{2}$, WEIPING LIU ${ }^{2}$, YANHUA BAI ${ }^{1}$ and YUMEI LAI ${ }^{1}$ \\ Departments of ${ }^{1}$ Pathology and ${ }^{2}$ Lymphoma, Key Laboratory of Carcinogenesis and Translational Research \\ (Ministry of Education/Beijing), Peking University Cancer Hospital and Institute, Beijing 100142, P.R. China
}

Received August 8, 2015; Accepted February 27, 2017

DOI: $10.3892 / \mathrm{ol} .2017 .6180$

\begin{abstract}
Anaplastic lymphoma kinase (ALK)-positive large B-cell lymphoma (LBCL) is a rare lymphoma subtype. The present study investigated a refractory nodal ALK-positive LBCL case in a 28-year-old Chinese male. It was initially misdiagnosed as ALK-positive anaplastic large cell lymphoma; however, the patient's lesions relapsed and spread widely following a short remission for chemotherapy and the patient succumbed to the disease 3 months' post-autologous stem cell transplantation; thus, a revision was performed. Histologically, the tumor cells exhibited a characteristic immunoblastic morphology with marked cellular pleomorphism. All lesions shared the same immunoprofiles, including granular cytoplasmic ALK staining patterns and a lack of cell lineage-associated markers, with the exception of cluster of differentiation (CD)45 and CD4. CD30 expression was revealed to be negative and CD138 staining was observed to be positive, additionally, cytokeratin was expressed aberrantly in a relapsed tumor biopsy. Fluorescence in situ hybridization studies demonstrated breakage and extra copies of the $A L K$ gene in $\geq 30 \%$ of cells. Final clarification was provided by the detection of immunoglobulin $\kappa$ locus $(I G K)$ gene rearrangement in clonality studies [but notimmunoglobulin heavy locus $(I G H)$ and immunoglobulin $\lambda$ locus $(I G L)$ genes]. This aggressive entity requires distinct modalities of standard treatment, and may be ignored owing to its rarity in routine pathology laboratories. BIOMED-2 polymerase chain reaction
\end{abstract}

Correspondence to: Dr Xianghong Li, Department of Pathology, Key Laboratory of Carcinogenesis and Translational Research (Ministry of Education/Beijing), Peking University Cancer Hospital and Institute, 52 Fucheng Road, Haidian, Beijing 100142, P.R. China E-mail: xhli72@163.com

Key words: anaplastic lymphoma kinase, B-cell lymphoma, cluster of differentiation 4 , cytokeratin, clonality assays, including for $I G H, I G K$ and $I G L$ genes, are essential for the detection of gene rearrangement.

\section{Introduction}

Anaplastic lymphoma kinase (ALK)-positive large B-cell lymphoma (LBCL) is a rare variant of diffuse large B-cell lymphoma (DLBCL) with an incidence of $\geq 1 \%$ of DLBCL (1). It was originally described by Delsol et al (2) in 1997, and only $<60$ cases have been identified to date (3-5). It is charac terized by a sinusoidal growth pattern and was composed of a monomorphic population of large immunoblast-like or plasmablast-like cells, marked ALK/epithelial membrane antigen (EMA) protein expression, lack of CD20 expression and an aggressive clinical course (3). Despite this subtype of lymphoma being recognized in the 2008 World Health Organization classi fication (1), its identification in routine pathology laboratories remains challenging, particularly in differential diagnosis among ALK-positive ALCL of null cell lineage and poorly differentiated anaplastic carcinoma (1). Recognition of this variant of DLBCL is important as the conventional therapy used for typical DLBCL is of limited efficacy in this disease phenotype. Novel front-line intensive chemotherapy regimens should be evaluated in this group of patients.

Cluster of differentiation (CD) 4 is normally a $\mathrm{T}$ helper cell-associated antigen, which is expressed in mature T-cell and natural killer cell lymphomas (1), but it has also been observed in certain ALK-positive LBCL cases (3). Pan-cytokeratin $(\mathrm{CK})$ is a useful biomarker for epithelial cells, which has been observed in certain lymphomas, including ALK-positive LBCLs $(3,6-8)$, and this may result in misdiagnosis. In the present study, a relapsed case of ALK-positive LBCL with unusual expression levels of CD4 and CK were observed, which appeared positive in the relapsed lesion.

\section{Case report}

Description of the case. A 28-year-old Chinese male presented with a submaxillary mass $4.0 \mathrm{~cm}$ in diameter in 

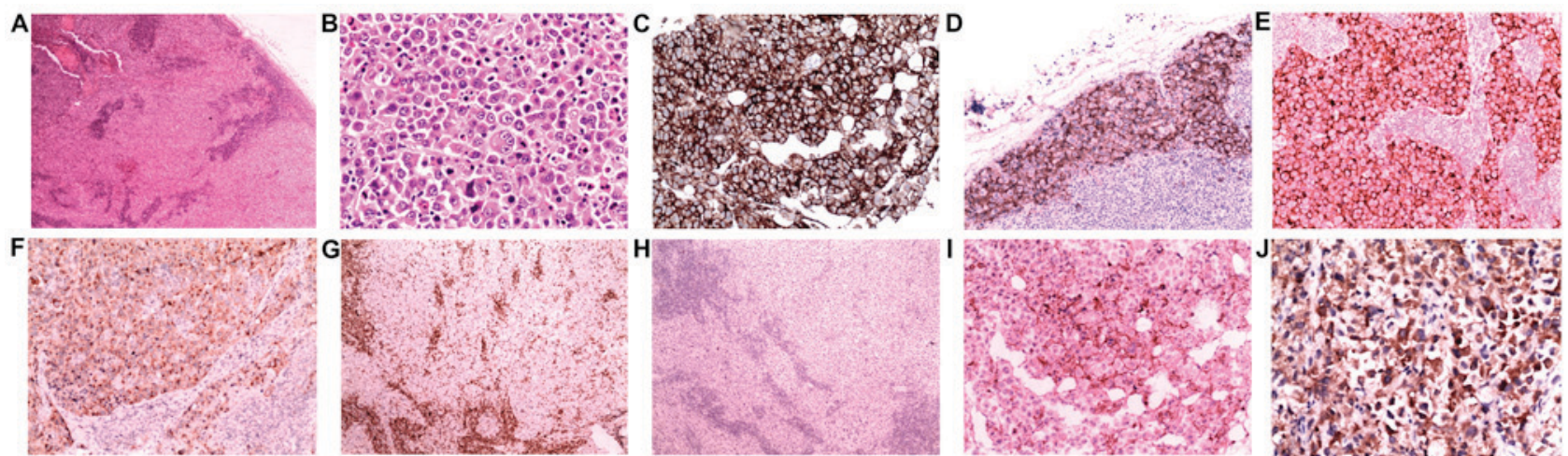

Figure 1. Lymph node biopsies reveal diffuse and sinus proliferation of large lymphocytes with immunoblastic features. (A) H\&E stain; magnification, $\mathrm{x} 40$. (B) H\&E stain;magnification, x400. The lymphoma cells were positive for (C) CD45, (D) CD138 and (E) epithelial membrane antigen, and they exhibited (F) granular cytoplasmic anaplastic lymphoma kinase positivity. Stains for (G) CD43 and (H) CD30 were negative. A subset of tumor cells was moderately positive for (I) CD4. (J) CytokeratinAE1/3 staining of neoplastic cells was positive in the last relapsed needle biopsy. (C and J) Immunoperoxidase stain; magnification, x400. (D, E, F, I) Immunoperoxidase stain; magnification, x200. (G and H) Immunoperoxidase stain; magnification, x40. H\&E, hematoxylin and eosin; $\mathrm{CD}$, cluster of differentiation.

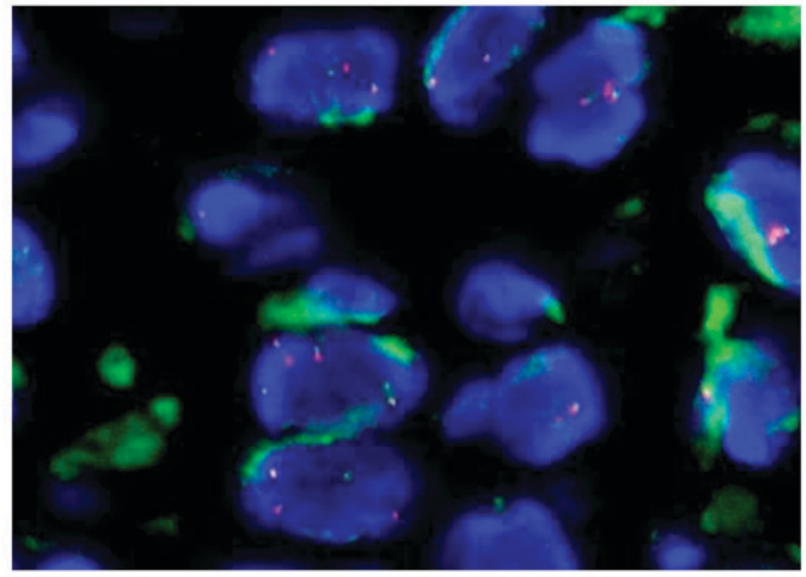

Figure 2. Representative metaphase fluorescence in situ hybridization in $A L K$-positive large B-cell lymphoma; magnification, $\mathrm{x} 1,000)$. The hybridization targets of the orange color probes and the green color probes are on opposite sides the breakpoint of the ALK gene. A two-color signal would represent a fusion signal in patients with native $A L K$, but they became two separated signals (one orange and one green) in this patient with ALK break-apart pattern. In addition, extra ( $>2)$ copies of the $A L K$ gene in single cell were also observed. ALK, anaplastic lymphoma kinase.

February 2011. The patient then suffered from a progressive enlargement of cervical nodes for 5 months without exhi biting any systemic symptoms. He received his treatment in the Peking University Cancer Hospital (Beijing, China), and a computed tomography (CT) scan revealed cervical, submaxillary and submentum lymphadenopathy. An excisional biopsy of the right cervical lymph node was performed prior to diagnosis as ALK-positive anaplastic large cell lymphoma (ALCL) and the patient's clinical stage was classified as IIB. The patient underwent 6 cycles of cyclophosphamide, doxorubicin, vincristine and prednisolone (CHOP). The restaging $\mathrm{CT}$ scan revealed complete remission following treatment. In May 2014, a solitary enlarged cervical lymph node was detected by a thorough examination and was surgically biopsied and it was again pathologically diagnosed as ALK-positive ALCL. A follow-up investigation was performed and the CT scan indicated that it was a progressive disease. The patient subsequently underwent 2 cycles of dexamethasone, ifosfamide, carboplatin and etoposide followed by autologous stem cell transplantation using cyclophosphamide, doxorubicin and vincristine treatment as a high-dose therapy regimen in January 2015. The disease relapsed again 6 weeks post-transplantation. A CT scan revealed a bulky mediastinal mass with axillary, supraclavicular, bilateral cervical and retroperitoneal lymphadenopathy, the patient's left breast and liver were also possibly involved. A core biopsy was performed, which revealed ALK-positive ALCL. The patient finally succumbed to the disease owing to respiratory failure in April 2015. A revision of the previous pathological diagnosis was performed as requested by the oncologist and the final diagnosis was ALK-positive LBLC with complex ALK gene rearrangements, CD4 continuously expressed and CK observed in the tumor specimen. These phenotypes were difficult for the pathologists to identify.

Histological and immunohistochemical (IHC) results. Paraffin-embedded samples from the two excisions and the final core biopsies were obtained and cut into 5- $\mu \mathrm{m}$ thick sections, then deparaffinized in xylene and rehydrated. The routine hematoxylin and eosin stains were then carried out for histological review under light microscope (Olympus X51; Olympus, Watford, UK), lymph node demonstrated a complete effacement of the normal structure by the diffuse proliferation of large neoplastic cells. Tumor cells proliferated in sheets and infiltrated sinuses (Fig. 1A) in certain regions. Atypical tumor cells were observed with immunoblastic features (round pale nuclei, large prominent nucleoli and abundant cytoplasm; Fig. 1B). A number of multinucleated cells were also presented, but the classical hallmark cell of ALCL was not observed.

The 4- $\mu \mathrm{m}$-thick formalin-fixed paraffin-embedded tumor tissue sections were used for all IHC staining, and the staining was performed using a BenchMark ULTRA automated IHC staining instrument and an ultraVIEW kit (Benchmark XT; Ventana Medical Systems, Tucson, AZ). All procedures including incubation time and temperature were performed according to the manufacture's protocol. 


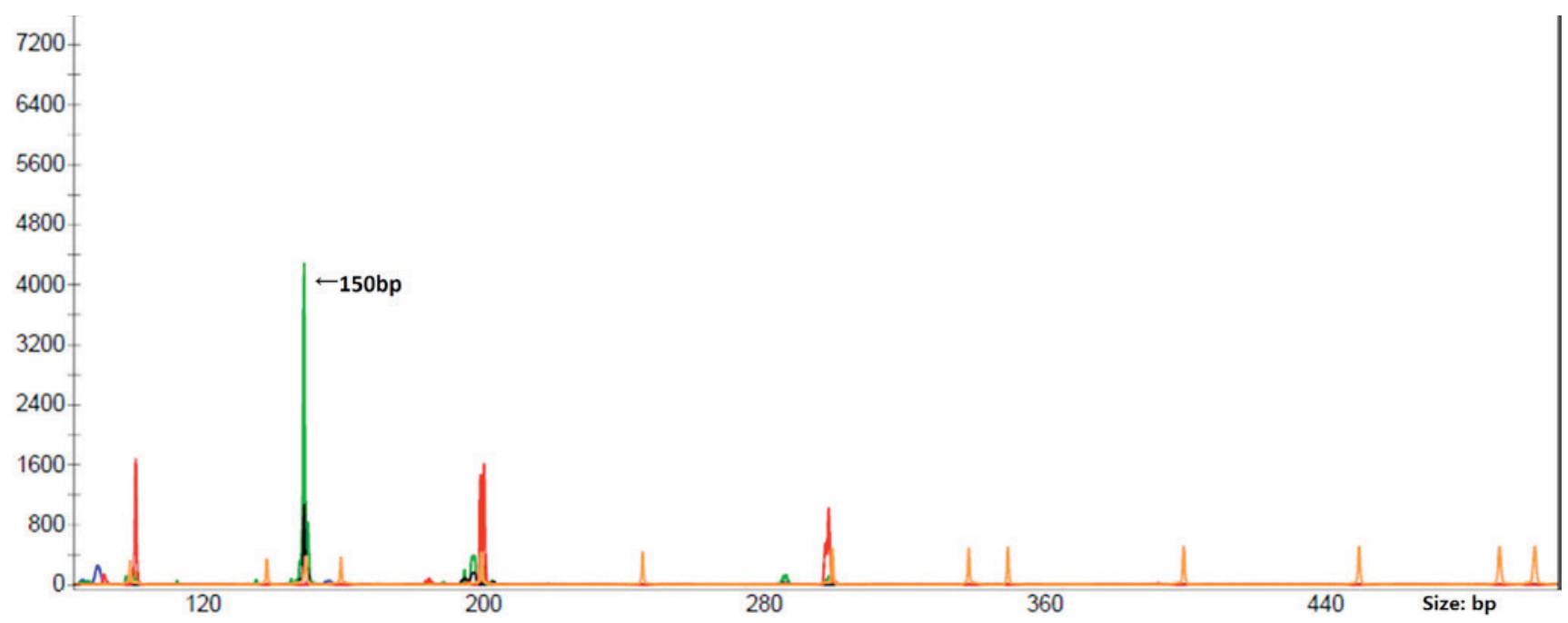

Figure 3. Clonal IGK locus gene rearrangement with polymerase chain reaction-based clonality investigation. The peak represented by the green line demonstrates the clonal rearrangement of variable $(\mathrm{V}) \kappa$-joining $(\mathrm{J}) \kappa($ detected in IGK tube A). IGK, immunoglobulin $\kappa$.

The primary antibodies applied in the biopsies were as following: CD20 (cat. no., GA60461-2; dilution, 1:100; Dako; Agilent Technologies, Santa Clara, CA, USA,), Bcl-6 (cat. no., IS62530-2; dilution, 1:100; Dako; Agilent Technologies), MUM-1 melanoma-associated antigen (mutated) 1 (cat. no., M725929-2; dilution, 1:100; Dako; Agilent Technologies), and CD10 (cat. no., CD10-270-L-CE; dilution, 1:50; Novocastra, Leica Biosystems, UK) was performed. CD30(cat. no., CD30-591-L-CE; dilution, 1:50; Novocastra), CD15(cat. no., IR06261-2; dilution, 1:100; Dako; Agilent Technologies), EMA (cat. no., M061329-2; dilution, 1:100; Dako; Agilent Technologies), ALK-1(cat. no., IR64161-2; dilution, 1:50; Dako; Agilent Technologies), CD45(cat. no., GA75161-2; dilution, 1:100; Dako; Agilent Technologies), CD43(cat. no., GA63661-2; dilution, 1:100; Dako; Agilent Technologies), CD3(cat. no., CD3-565-L-CE; dilution, 1:100; Novocastra), CD4(cat. no., IS64930-2, dilution, 1:100, Dako, Agilent Technologies), paired box 5/PAX5(cat. no., PA1-109, dilution, 1:100, Thermo Fisher, Rockford, USA), Ki67(cat. no., GA62661-2; dilution, 1:100; Dako; Agilent Technologies), B-cell lymphoma 2/bcl2 (cat. no., M088729-2; dilution, 1:50; Dako; Agilent Technologies), CK (ca. no., M351501-2; dilution, 1:100; Dako; Agilent Technologies) and cam5.2 (cat. no., 349205; dilution, 1:100; BD Biosciences, San Jose, CA, USA); and CD138 (cat. no., GA64261-2; dilution, 1:100; Dako; Agilent Technologies) and CD79a (cat. no., M705029-2; dilution, 1:100; Dako; Agilent Technologies) were added during the revision. The results of IHC analysis revealed that almost all the large neoplastic cells were markedly positive for CD45, CD138 and EMA (Fig. 1C-E). ALK-1 staining was markedly positive in neoplastic cells with a granular cytoplasmic pattern (Fig. 1F) and MUM1 was expressed partially in tumor cells. Immunostaining of Ki67 demonstrated a proliferation index of $\sim 45 \%$. Neoplastic cells were negative for CD43 (Fig. 1G), CD30 (Fig. 1H), CD20, CD79a, CD15, PAX5 and CD3 in the patient's malignancies. Subsets of neoplastic cells were positive for CD4 (Fig. 1I). CK (Fig. 1J) was negative in excision biopsies, but was moderately positive in the needle biopsy.
Epstein-Barr virus (EBV) in situ hybridization. Tissue sections were hybridized as described (9) with a biotin-labeled nucleic acid probe (Pan Path, Budel, The Netherlands) to determine the existence of EBV. Specimens with EBER nuclear expression would indicate latent EBV infection by the presence of RNA which was complementary to EBV. However, no EBV infection was detected by in situ hybridization in the present case.

Fluorescence in situ hybridization (FISH). Interphase FISH analysis was performed during the needle biopsy using the VysisLSI ALK Dual Color Break Apart Rearrangement Probe (Abbott Pharmaceutical Co., Ltd., Lake Bluff, IL, USA), which contains loci flanking the typical $A L K$ gene breakpoint at 2p23.3 in order to detect suspected $A L K$ gene rearrangement. The FISH slides were reviewed and under a fluorescence microscope (BX60, Olympus), and images were captured via camera and molecular genetics software (Cytovision Genus v7.1; Leica Microsystems, Ltd, Milton Keynes, UK). The break-apart pattern is a fusion signal (native $A L K$ ) and became two separated signals (one orange and one green). The distance between two separated signals was estimated using at least twice the largest signal size and positive cases were defined as $\geq 15$ cells with a break-apart pattern in 100 tumor cells. ALK gene translocation in this case was demonstrated as positive, with 1 or 2 extra copies of $A L K$ gene presented (Fig. 2) in $\geq 30 \%$ of cells.

Clonality studies. The polymerase chain reaction (PCR) was performed using a multiplex PCR of European BIOMED-2 assays (Yuanqi Bio, Shanghai, China). Primers for detecting clonally rearranged immunoglobulin $(\mathrm{Ig})$ were set in 8 multiplex PCR tubes: A total of 5 Ig heavy locus ( $I G H$; including3 variable-joining domains and 2diversity-joining domains), 2 Ig $\kappa$ locus $(I G K)$ and $1 \operatorname{Ig} \lambda$ locus $(I G L)$. T-cell receptor $(T C R)$ gene rearrangement was performed using primers in 6 multiplex PCR tubes: A total of 3 TCR $\beta, 2$ TCR $\gamma$ and 1 TCR $\delta$. The clonal rearrangement was detected only in the A tube of the $I G K$ gene (Fig. 3). No IGH, IGL or TCR gene rearrangements were detected. 


\section{Discussion}

The results of the present relapsed case tumor morphology and immunophenotype investigations from the routine pathological department led to differential diagnoses of hematopoietic and non-hematopoietic neoplasms, in particular ALK positive-ALCL and metastatic carcinoma. First, CK expression may mislead to a poorly differentiated carcinoma, but marked expression of CD45 (LCA) would easily exclude this misdiagnosis. Secondly, the IHC results demonstrated that tumor cells were positive for ALK-1 and EMA, but negative for commonly used T- and B-cell markers (CD20, CD79a, CD3 and CD43), with the exception of a low expression level of CD4, which is generally considered a T helper cell marker and is normally expressed in ALCL. Thus, the diagnosis of ALK-positive ALCL of null cell lineage was suggested to be the most suitable, although CD30 staining was repeated twice and revealed negative results at the beginning.

The initial misdiagnosis of the present study may also be attributed to not identifying the ALK-positive LBCL entity owing to its rarity. Further plasma cell differentiated markers and clonality studies for T- or B-cell origin would be useful to confirm a correct diagnosis. The additional IHC staining for CD138 was positive, which was consistent with the reported positive ratio of CD138, EMA, CD20 and CD79a in ALK-positive LBCL, which were 100,97.6, 11 and 18\%, respectively (5). Expression of the T-cell marker CD4 was identified in between 37.8 and $40 \%$ of ALK-positive LBCL cases $(3,5)$, which may be the reason for the confusion over the origin of the tumor cell. The final definite diagnosis of the present case was based on the clonality analysis. However, as indicated in a previous study, PCR clonal analyses for IGH were identified in a majority of patients demonstrating rearrangement genes (5), but there was also a similar case reported by with rearrangement if $I G H$ genes were solely detected (10), thus making it more difficult to reach a precise diagnosis, particularly in demonstrating no $\mathrm{T}$ - nor B-cell immunophenotypes. Thus, the BIOMED-2 PCR assays for $I G K$ and $I G L$ gene rearrangements are essential for B-cell clonality analysis in this subtype of lymphoma, and a previous study also revealed $I G K$ gene rearrangements (10); however, there appeared to be no $I G L$ gene rearrangements reported in the ALK-positive LBCL cases.

The majority of the ALK fusion protein in ALK-positive LBCL came from cytogenetic fusion of the $A L K$ gene at chromosome 2p23 and clathrin heavy-chain (CLTC) gene at chromosome 17q23 $(1,3)$. The nucleophosmin gene at chromosome $5 \mathrm{q} 35$ was the most common partner observed in ALK positive ALCL (7,11-16). The granular cytoplasmic ALK staining pattern demonstrated in the present case was similar to the correlation with the CLTC-ALK fusion observed in a previous study by Gascoyne et al (17) and De Paepe et al (18) in 2003.The present case also exhibited one or two extra ALK copies demonstrating the high genetic instability detected using FISH, which is characteristic of ALK-positive LBCLs (16). However, ALK protein expression and genomic alteration was not useful for the differential diagnosis between ALK positive ALCL and ALK positive LBCL.

To the best of our knowledge, CK expression has been identified in $<5$ ALK positive LBCL cases previously $(5,8)$, whereas CD4 expressed has been observed in $>5$ previous ALK positive LBCL cases. The aberrant expression levels of CK and CD4 in ALK-positive LBCL are rare. Only the final relapsed malignancy in the present case was positive for $\mathrm{CK}$. It was previously reported that CLTC was located at chromosomal locus $17 \mathrm{q} 23$ and a cluster of human keratin-associated protein genes were revealed to be embedded at chromosomal locus 17q12-21 (19), which is in proximity to CLTC. This suggested that the translocation and instability of the CLTC gene may induce an incidental activation of the keratin-associated gene and CK expression. Further investigation of this is required.

The recognition of ALK-positive DLBCL is also clinically important as the majority of patients follow a more aggressive disease course and unfavorable prognosis (3). ALK-positive LBCLs are unlikely to respond favorably to the current standard CHOP therapy for ALCL. They require distinct modalities of treatment and candidates for novel treatment approaches, with or without autologous stem cell transplantation; novel biological agents including anti-CD138 or anti-ALK monoclonal antibodies or other immune conjugates $(20,21)$ may provide future treatment strategies. The aggressive clinical course of the present case further confirmed the diagnosis of ALK-positive LBCL, an aggressive form of DLBCL with ectopic ALK expression due to CLTC-ALK gene fusion (3).

In conclusion, ALK-positive large B-cell lymphoma expressed cluster of differentiation 4 and cytokeratin are extremely rare and easily be misdiagnosed, thus standardizedBIOMED-2 PCR assays that encompass the IGH,IGL and, particularly, IGK gene rearrangements are essential in B-cell gene clonality analysis for this subtype of lymphoma.

\section{Acknowledgements}

The authors thank all their colleagues who participated in the analyses and revision of the present case.

\section{References}

1. Swerdlow S.H Campo, E, Harris N.L, Jaffe E.S, Pileri S.A, Stein H, Thiele J and Vardiman J.W: WHO classification tumours of haematopoietic and lymphoid tissues. 4th edition. Lyon, France: IARC Press pp244-255, 2008.

2. Delsol G, Lamant L, Mariamé B, Pulford K, Dastugue N, Brousset P, Rigal-Huguet F, al Saati T, Cerretti DP, Morris SW and Mason DY: A new subtype of large B-cell lymphoma expressing the ALK kinase and lacking the 2; 5 translocation. Blood 89: 1483-1490, 1997.

3. Laurent C, Do C, Gascoyne RD, Lamant L, Ysebaert L, Laurent G, Delsol G and Brousset P: Anaplastic lymphoma kinase-positive diffuse large B-cell lymphoma: A rare clinicopathologic entity with poor prognosis. J Clin Oncol 27: 4211-4216, 2009.

4. Huang RF, CHEN G, GONG LP and LU LL: Pathologic and molecular genetic study of anaplastic lymphoma kinase-positive large B-cell lymphoma. Zhonghua Bing Li Xue Za Zhi 40: 169-172, 2011 (In Chinese).

5. Yu H, Huang J, Sun J, Wang C, Lin M and Li H: Anaplastic lymphoma kinase-positive large B-cell lymphoma: A potential diagnostic pitfall. Indian J Pathol Microbiol 58: 241-245, 2015.

6. Gustmann C, Altmannsberger M, Osborn M, Griesser H and Feller AC: Cytokeratin expression and vimentin content in large cell anaplastic lymphomasand other non-Hodgkin's lymphomas. Am J Pathol 138: 1413-1422, 1991.

7. Zhang Q, Ming J, Zhang S, Li B, Han X and Qiu X: Cytokeratin positivity in anaplastic large cell lymphoma: A potential diagnostic pitfall in misdiagnosis of metastatic carcinoma. Int J Clin Exp Pathol 6: 798-801,2013. 
8. Nguyen TT, Kreisel FH, Frater JL and Bartlett NL: Anaplastic large cell lymphoma with aberrant expression of multiple cytokeratins masquerading as metastatic carcinoma of unknown primary. J Clin Oncol 31: e443-e445, 2013.

9. Shi Y and Li X: Clinicopathologic features and association with Epstein-Barr virus infection in 235 cases of Hodgkin lymphoma from northern China. Zhonghua Bing Li Xue Za Zhi 44: 84-89, 2015 (In Chinese).

10. Lin SY, Chuang SS, JhuangJY, Sakamoto K, Takeuchi K, Bahrami A and Tsai CC: ALK positive large B-cell lymphoma with a massive neutrophilic infiltrate: Report of a case mimicking epithelioid inflammatory myofibroblastic tumour. J Clin Pathol 68: 496-498, 2015.

11. Reichard KK, McKenna RW and Kroft SH: ALK-positive diffuse large B cell lymphoma: Report of four cases and review of the literature. Mod Pathol 20: 310-319, 2007.

12. Rudzki Z, Rucińska M, Jurczak W, Skotnicki AB, Maramorosz-Kurianowicz M, Mruk A, Piróg K, Utych G, Bodzioch P, Srebro-Stariczyk M, et al: ALK-positive diffuse largeB-cell lymphoma: Two more cases and a brief literature review. Pol J Pathol 56: 37-45, 2005.

13. Onciu M, Behm FG, Downing JR, Shurtleff SA, Raimondi SC Ma Z, Morris SW, Kennedy W, Jones SC and Sandlund JT: ALK-positive plasmablastic B-cell lymphoma with expression of the NPM-ALK fusion transcript: Report of 2 cases. Blood 102 2642-2644, 2003.

14. Adam P, Katzenberger T, Seeberger H, Gattenlöhner S, Wolf J, Steinlein C, Schmid M, Müller-Hermelink HK and Ott G: A case of a diffuse large B-cell lymphoma of plasmablastic type associated with the $\mathrm{t}(2 ; 5)(\mathrm{p} 23 ; \mathrm{q} 35)$ chromosome translocation. Am J Surg Patho 27: 1473-1476, 2003.
15. Lee HW, Kim K, Kim W and Ko YH: ALK-positive diffuse large B-cell lymphoma: Report of three cases. Hematol Oncol 26 : 108-113, 2008

16. Shi M, Miron PM, Hutchinson L, Woda BA, Nath R, Cerny J and Yu H: Anaplastic lymphoma kinase-positive large B-cell lymphoma with complex karyotype and novel ALK gene rearrangements. Hum Pathol 42: 1562-1567, 2011.

17. Gascoyne RD, Lamant L, Martin-Subero JI, Lestou VS, Harris NL, Müller-Hermelink HK, Seymour JF, Campbell LJ, Horsman DE, Auvigne I, et al: ALK-positive diffuse large B-cell lymphoma is associated with Clathrin-ALK rearrangements: Report of 6 cases. Blood 102: 2568-2573, 2003.

18. De Paepe P, Baens M, van Krieken H, Verhasselt B, Stul M, Simons A, Poppe B, Laureys G, Brons P, Vandenberghe P, et al: ALK activation by the CLTC-ALK fusion is a recurrent eventin in large B-cell lymphoma. Blood 102: 2638-2641, 2003.

19. Rogers MA, Langbein L, Winter H, Ehmann C, Praetzel S, Korn B and Schweizer J: Characterization of a cluster of human high/ultrahigh sulfur keratin-associated protein genes embedded in the type I keratin gene domain on chromosome 17q12-21. J Biol Chem 276: 19440-19451, 2001.

20. Castillo JJ, Chavez JC, Hernandez-Ilizaliturri FJ and Montes-Moreno S: CD20-negative diffuse large B-cell lymphomas: Biology and emerging therapeutic options. Expert Rev Hematol 8: 343-354, 2015.

21. Roskoski R Jr: Anaplastic lymphoma kinase (ALK): Structure, oncogenic activation, and pharmacological inhibition. Pharmacol Res 68: 68-94, 2013. 